\title{
MGMT expression predicts response to temozolomide in pancreatic neuroendocrine tumors
}

\author{
J Cros',2,*, O Hentic 3,*, V Rebours2,3, M Zappa4, N Gille1, N Theou-Anton5, \\ D Vernerey6, F Maire³, P Lévy2,3, P Bedossa1,2, V Paradis1,2, P Hammel2,7, \\ P Ruszniewski2,3 and A Couvelard2,8 \\ 1Department of Pathology, AP-HP, DHU UNITY, Beaujon University Hospital, Clichy, France \\ 2U1149 - University Paris Diderot, Paris, France \\ ${ }^{3}$ Department of Gastroenterology and Pancreatology, AP-HP, DHU UNITY, Beaujon University Hospital, Clichy, France \\ ${ }^{4}$ Department of Radiology, AP-HP, DHU UNITY, Beaujon Hospital, Clichy, France \\ ${ }^{5}$ Department of Somatic Genetic, AP-HP, DHU UNITY, Bichat University Hospital, Paris, France \\ ${ }^{6}$ Methodology and Quality of Life in Oncology Unit (EA 3181), University Hospital of Besançon, Besançon, France \\ 7Department of Digestive Oncology, AP-HP, DHU UNITY, Beaujon University Hospital, Clichy, France \\ ${ }^{8}$ Department of Pathology, AP-HP, DHU UNITY, Bichat University Hospital, Paris, France \\ ${ }^{*}(\mathrm{~J}$ Cros and O Hentic contributed equally to this work)
}

Correspondence should be addressed to A Couvelard Email anne.couvelard@bch.aphp.fr

\begin{abstract}
Temozolomide (TEM) showed encouraging results in well-differentiated pancreatic neuroendocrine tumors (WDPNETs). Low ${ }^{6}$-methylguanine-DNA methyltransferase (MGMT) expression and MGMT promoter methylation within tumors correlate with a better outcome under TEM-based chemotherapy in glioblastoma. We aimed to assess whether MGMT expression and MGMT promoter methylation could help predict the efficacy of TEM-based chemotherapy in patients with WDPNET. Consecutive patients with progressive WDPNET and/or liver involvement over $50 \%$ who received TEM between 2006 and 2012 were retrospectively studied. Tumor response was assessed according to Response Evaluation Criteria in Solid Tumors (RECIST) 1.1 guidelines. Nuclear expression of MGMT was assessed by immunochemistry (H-score, 0-300) and MGMT promoter methylation by pyrosequencing. Forty-three patients ( 21 men, 58 years (27-84)) with grade 1 WDPNET $(n=6)$ or $2(n=36)$ were analyzed. Objective response, stable disease, and progression rates were seen in 17 patients (39.5\%), 18 patients $(41.9 \%)$, and 8 patients (18.6\%), respectively. Low MGMT expression $(\leq 50)$ was associated with radiological objective response $(P=0.04)$ and better progression-free survival (PFS) ( $\mathrm{HR}=0.35(0.15-0.81), P=0.01)$. Disease control rate at 18 months of treatment remained satisfying with an MGMT score up to 100 (74\%) but dropped with a higher expression. High MGMT promoter methylation was associated with a low MGMT expression and longer PFS (HR $=0.37(0.29-1.08), P=0.05)$. Low MGMT score $(\leq 50)$ appears to predict an objective tumor response, whereas an intermediate MGMT score (50-100) seems to be associated with prolonged stable disease.
\end{abstract}

\section{Key Words}

- temozolomide

- MGMT

- pancreatic neuroendocrine tumors

- methylation
C 2016 Society for Endocrinology Printed in Great Britain
Published by Bioscientifica Ltd.
Endocrine-Related Cancer

(2016) 23, 625-633 


\section{Introduction}

Evidences suggesting the efficacy of temozolomide (TEM), a DNA-alkylating agent, in advanced pancreatic neuroendocrine tumors (PNETs) are accumulating (Kulke et al. 2006, Ekeblad et al. 2007, Strosberg et al. 2011, Chan et al. 2012, Olsen et al. 2012, Fine et al. 2013). With the availability of other systemic treatments such as targeted therapies, the need for companion biomarkers is greater than ever. There is a strong biological rational to assess the $\mathrm{O}^{6}$-methylguanine-DNA methyltransferase (MGMT) function before TEM-based treatment (Zhang et al. 2011). This suicide enzyme removes alkyl group from the $O^{6}$ position on guanine bases, one of the main sites of TEMinduced DNA damage. Without MGMT, altered guanine mispairs with thymine instead of cytosine during DNA replication and is not properly repaired by the DNA mismatch repair (MMR) system. Futile repair cycles cause DNA replication fork collapse, cell cycle arrest, and ultimately apoptosis (Zhang et al. 2011). Therefore, TEM-induced antitumor activity is believed to rely on both low MGMT level and a functional MMR system. Glioblastomas were the first tumors investigated, but now data are confirming the value of this enzyme to predict the efficacy of TEM in other tumor types (Hegi et al. 2005, Schraml et al. 2012). There is no consensus on how to best assess MGMT. Methylation of MGMT promoter was associated with a prolonged overall survival in patients with glioblastoma receiving TEM (Hegi et al. 2005). Among the available techniques to assess MGMT promoter methylation, pyrosequencing appears to be the most robust (Karayan-Tapon et al. 2010, Quillien et al. 2012). Similar findings regarding the predictive value of MGMT promoter methylation were suggested in PNETs (Schmitt et al. 2014, Walter et al. 2015). In addition, methylation of the MGMT promoter appears to have a prognostic value in neuroendocrine tumor (NET) independently of TEM treatment. MGMT promoter methylation is associated with a wide methylation of the genome, which introduces a potential bias in the assessment of the predictive value of this biomarker (Schmitt et al. 2014, Walter et al. 2015).

Assessment of MGMT expression by immunochemistry, a cost-effective method, has also been proposed, but it did not seem to correlate well with promoter methylation (Uno et al. 2011). Since then, various methylation-independent mechanisms of MGMT expression have been described, explaining this discrepancy (Lavon et al. 2007, Kitange et al. 2012, Kohsaka et al. 2012, Kreth et al. 2013). Finally, it was suggested that MGMT expression assessed by immunochemistry could be a good predictor of response to TEM-based treatment in patients with PNET, but the cohorts reported until yet were small and heterogeneous (Ekeblad et al. 2007, Kulke et al. 2009, Schmitt et al. 2014, Walter et al. 2015).

In this study, we aimed to evaluate retrospectively the predictive value on treatment efficacy (i.e., radiological response rate, progression-free survival (PFS)) of MGMT in a large and homogeneous cohort of patients with well-characterized PNETs treated by TEM in a single tertiary center. MGMT expression was assessed by immunochemistry on FFPE samples and MGMT promoter methylation by pyrosequencing.

\section{Patients and methods}

\section{Patients and treatment}

The study was approved by the ethical review board of our institution (IRB 00006477-15.014). All consecutive patients with histologically proven, locally advanced or metastatic WDPNETs treated with TEM, alone or in combination with capecitabine, in Beaujon University Hospital between February 2005 and June 2012 were selected. Clinical and pathological characteristics of the patients and their tumors were collected from the surgical, clinical, and pathological files. Tumors were classified according to the 2010 WHO classification and the ENETS grading system (Rindi et al. 2006). TEMbased regimens were proposed regardless of previous therapeutic line in case of progression of metastases according to Response Evaluation Criteria in Solid Tumors (RECIST) guidelines on two consecutive imaging procedures performed at 6-month intervals and/or when liver burden was $>50 \%$ (Skinazi et al. 1996, Durante et al. 2009, Pavel et al. 2012). Patients received TEM alone or in combination with capecitabine. TEM was administrated orally for five consecutive days every 28 days at $150 \mathrm{mg} / \mathrm{m}^{2}$ once daily (days $1-5$ ) for the first cycle and $200 \mathrm{mg} / \mathrm{m}^{2}$ in the subsequent cycles if no major hematological toxicity occurred. In the combination, capecitabine was administrated orally every 28 days at $750 \mathrm{mg} / \mathrm{m}^{2}$ twice daily (days 1-14) and TEM as described above (days 10-14).

\section{Radiological evaluation}

Tumor response rate was assessed by CT scan at 3-month intervals according to RECIST 1.1 guidelines (Eisenhauer

Published by Bioscientifica Ltd 
et al. 2009). The best response was considered among all response assessments. Evaluation procedures were performed ahead of schedule if patient general condition deteriorated or severe toxicity occurred. PFS was calculated from the first day of the first cycle of chemotherapy cycle until clinical and/or radiological progression.

\section{MGMT immunohistochemical evaluation}

Paraffin-embedded sections of $4 \mu \mathrm{m}$ were used for immunohistochemistry. MGMT expression was assessed on 24 samples from the primary pancreatic tumor and 19 samples from liver metastasis (biopsy or surgical specimens). For surgical specimens, one representative block of the tumor was selected. Samples were only selected if they were collected before TEM initiation. For antigen retrieval, sections were subjected to pretreatment in EDTA buffer $\mathrm{pH} 9$ at high temperature. Immunohistochemistry was done using a Bond MAX autostainer (Leica). Sections were incubated with a mouse monoclonal MGMT antibody (clone MT3.1 (Thermo Scientific), diluted at $1 / 25$, and incubated for $20 \mathrm{~min}$. The reaction product was revealed with the DAB chromogen for $10 \mathrm{~min}$. Tumors without an internal control (endothelial cell for instance) were not evaluated. MGMT expression was assessed on whole slide using a score based on nuclear staining intensity (0-3) multiplied by the percentage of stained cells (0-100\%). The score ranged from 0 to 300. Examples of different MGMT scores are represented in Fig. 1. The slides were independently reviewed by two pathologists (J C and A C), blinded to the clinical outcome. The agreement was assessed by computing the chance-corrected Cohen's $\kappa$ score using three MGMT categories $(\leq 50,>50$ to $\leq 100$, $>100$ ) (Cohen 1968).

\section{DNA extraction, MGMT promoter methylation and MSI status determination}

DNA was isolated from FFPE samples. Microdissection was performed if the percentage of tumor cells was below 80\%. DNA was extracted using the QIAamp DNA Mini Kit (Qiagen). Bisulfite conversion was achieved with the EpiTech Bisulfite Kit (Qiagen). Pyrosequencing was performed with the PyroMark Q24 MGMT kit (Qiagen) on a PyroMark Q24 system (Qiagen). Templates for pyrosequencing were obtained by amplifying bisulfitetreated DNA with primers that are biotinylated for template strands. The biotinylated PCR products were then immobilized on streptavidin-coated Sepharose beads (GE Healthcare), and the single-stranded DNA templates were analyzed in the pyrosequencing instrument. For each $\mathrm{CpG}$ island tested, a mean result was calculated. For data analysis, the average percentage of the four CpGs was determined as well as the results of each tested CpG. According to the literature, a threshold of 7\% (mean of the four CpGs) was used to classify the patients in 'low' and 'high' promoter methylation groups (Quillien et al. 2012).

\section{Molecular analysis of microsatellite instability}

According to revised Bethesda guidelines, MSI analysis was performed with five consensus panel markers (BAT25, BAT26, NR21, NR22, NR24) (Wong et al. 2006). Techniques are routinely made with negative (normal colonic tissues) and positive (colon adenocarcinomas exhibiting the five positive markers cited above) controls. The GeneMapper software v4.0 allows the analysis of MSI marker migration. An MSI was considered to be present if at least three markers displayed instability (RER-positive and MSI-positive phenotype). When one or two markers were modified, the result was considered as MSI-negative phenotype.

\section{Statistical analysis}

General characteristics were expressed as median and range or percentages. Comparisons of variables were performed using the Kruskal-Wallis test for continuous data and the $\chi^{2}$ test or Fisher's exact test for categorical data. For survival analysis, the primary point used was the date of TEM initiation. PFS of the whole cohort was estimated by using the Kaplan-Meier method. Survival curves were compared with the log-rank test.
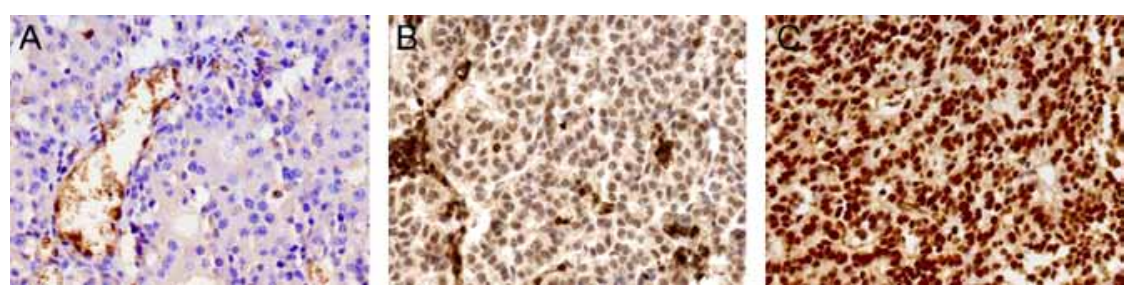

Figure 1

Examples of MGMT staining and corresponding MGMT score in WPDNET. Left panel: No nuclear staining (MGMT score $=0$ ); middle panel: weak nuclear staining (intensity 1 ) in $100 \%$ of the cells (MGMT score $=100 \times 1=100$ ); right panel: strong nuclear staining (intensity 3 ) in $100 \%$ of the cells (MGMT score $=100 \times 3=300$ ). 
Table 1 Patients characteristics and best radiological tumor response.

\begin{tabular}{lcc}
\hline Patients characteristics $(n=43)$ & $\boldsymbol{n}(\%)$ \\
\hline Sex (female) & & $22(51.2)$ \\
Age (mean) & & \\
Grade & & $(14)$ \\
G1 & $36(83.7)$ \\
G2 & $12(27.9)$ \\
G2 Ki-67>10\% & $1(2.3)$ \\
G3 & \\
Treatment(s) prior to TEM & $9(20.9)$ \\
None & $6(14.0)$ \\
Surgery alone & \\
Chemotherapy/targeted therapy & $15(34.9)$ \\
One line & $10(23.3)$ \\
Two lines & $3(7.0)$ \\
$\quad$ More than two lines & \\
Type of TEM treatment & $28(65.1)$ \\
TEM alone & $15(34.9)$ \\
TEM+capecitabine & \\
Tumor status at TEM initiation & $32(74.4)$ \\
Progression & $5(11.6)$ \\
Stable & $6(14.0)$ \\
Unknown & \\
Best morphological response (RECIST) & $8(18.6)$ \\
Progression & $18(41.9)$ \\
Stability & $17(39.5)$ \\
Objective response &
\end{tabular}

Several thresholds of the MGMT score were tested before define the more relevant value to predict the objective response to TEM chemotherapy. Survival analyses were performed according to the select threshold and the MGMT promoter methylation status. Data were analyzed with the SAS 9.1 statistical software for Windows (SAS Institute, Cary, NC, USA). All statistical tests were two sided. The critical level of statistical significance was set at $P<0.05$.

\section{Results}

\section{Patient characteristics and treatment response}

Forty-three patients with WDPNETs treated with TEM $(n=28)$ or TEM-CAP $(n=15)$ were studied. Patient's characteristics are summarized in Table 1 . Thirty-six patients $(83.7 \%)$ had a grade 2 WDPNET, and out of these, 12 had a tumor with a proliferation index (MIB-1/Ki-67) above $10 \%$. One patient had a well-differentiated grade 3 tumor (Ki-67=40\%). Five patients had a genetically proven MEN1 syndrome.

Thirty-two (74\%) patients were progressive at TEM initiation and five were stable. Tumor control was obtained in 35 patients $(81.4 \%)$ with 17 patients $(39.5 \%)$ displaying an objective response. Among the 32 patients whose tumor was progressive at TEM initiation, tumor control could be obtained in 24 of them (75\%) with 10 objective responses (31.2\%) and 14 stabilizations (43.7\%). The best radiological response according to the status at TEM initiation is presented in Supplementary Fig. 1, see section on supplementary data given at the end of this article. The median PFS was 20 months (range: 2-43).

\section{Predictive value of MGMT immunohistochemical expression}

WDPNETs were often deficient for MGMT, but the range of expression was large (median expression 5, range: $0-300)$. Thirty-three patients had an MGMT score $<100$ and seven had a score comprised between 100 and 200 . The agreement between the MGMT scores assessed by two pathologists was excellent $\left(\kappa=0.85, P<10^{-4}\right)$.

The median MGMT score was significantly lower in patients who had an objective response with TEM-based chemotherapy $(P=0.001)$ (Table 2$)$. MGMT scores in these patients were low with a narrow range (0-60). We, therefore, tested if a low threshold close to the higher limit in the objective response group, that is, MGMT score $\leq 50$, could be predictive of objective response. The number of patients with an MGMT score $\leq 50$ was significantly higher in the objective response group $(P=0.04)$ (Table 2$)$. We then tested if this threshold of 50 was associated with the PFS under TEM-based treatment. Patients with an MGMT score $\leq 50$ did have a longer PFS (HR: 0.35 (0.15-0.81), $P=0.01)$. We then assessed if an MGMT score $\leq 100$ was

Table 2 Predictive value of immunohistochemical expression of MGMT on radiological response.

\begin{tabular}{|c|c|c|c|c|}
\hline & \multicolumn{3}{|c|}{ Best radiological response } & \multirow[b]{2}{*}{$\boldsymbol{P}$} \\
\hline & $P$ & $\mathrm{~S}$ & OR & \\
\hline Median MGMT score (range) & $15(0-250)$ & $80(0-300)$ & $0(0-60)$ & 0.001 \\
\hline \multicolumn{5}{|l|}{ MGMT score } \\
\hline$\leq 50$ (30 patients) & $6 / 30(20 \%)$ & $9 / 30(30 \%)$ & $15 / 30(50 \%)$ & \\
\hline >50 (13 patients) & $2 / 13(15 \%)$ & $9 / 13(70 \%)$ & $2 / 13(15 \%)$ & 0.04 \\
\hline
\end{tabular}

Radiological response was assessed according to RECIST 1.1 guidelines.

OR, objective response; P, progression; S, stabilization. 


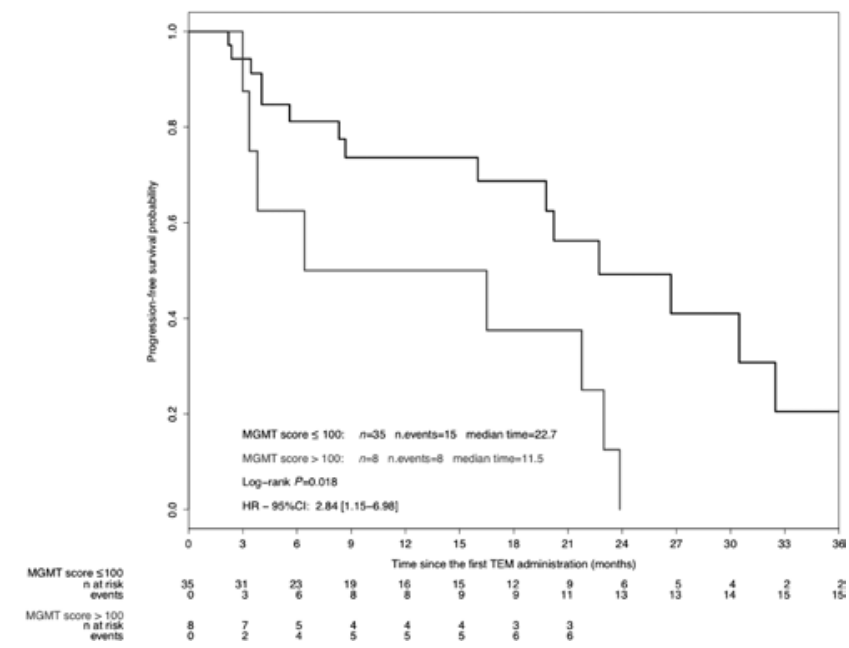

Figure 2

PFS in patients receiving TEM-based therapy for WDPNET with an MGMT immunohistochemical score above or below 100.

still associated with a better PFS. Indeed, patient with an intermediate MGMT score (100) had a longer PFS (HR: 0.37 (0.16-0.88), $P=0.02$ ) (Fig. 2).

Previous published studies used a low cutoff of 0 , 5 , or $10 \%$ of MGMT-expressing cells to dichotomize tumors, and the intensity of staining was not taken into account (Ekeblad et al. 2007, Kulke et al. 2009, Schmitt et al. 2014, Walter et al. 2015). This would translate assuming the maximum intensity of 3 to a cutoff score of 0,15 and 30. We, therefore, assess the predictive value of these cutoffs on PFS (Supplementary Fig. 2A, B, and C). With a very stringent cutoff of 0 , the MGMT expression was not associated with PFS (HR: 0.72 (0.32-1.66), $P=0.58)$. Raising the cutoff resulted in an association between MGMT expression and a shorter PFS (HR: 0.28
(0.11-0.69), $P=0.006$ for 15 and $\mathrm{HR}=0.25(0.10-0.66)$, $P=0.005$ for 30$)$.

In contrast to the use of a single cutoff point that might result in loss of information, the continuous MGMT expression scoring method reflects the wide range of expression seen in tumors. We, therefore, assessed how the proportion of patients with an objective response or a prolonged stabilization varies with increasing MGMT threshold. The MGMT score was known before treatment initiation for the last 10 patients selected in this study (i.e., they all had a low MGMT score). As this could introduce a bias in the study of the radiological response and tumor control according to MGMT expression (addition of selected patients with low MGMT), they were, therefore, excluded for the analyses presented below.

MGMT predictive value of radiological objective response We compared the number of objective response according to the MGMT score in the group of 33 patients with unknown MGMT score before TEM treatment (Fig. 3). The median MGMT score was lower in the objective response (OR) group (Fig. 3A). Thirteen out of the fourteen patients that presented an objective response had an MGMT score $\leq 50$. The remaining patient had an MGMT score of 60 . No patient with a score above 100 presented an objective response (Fig. 3B). Of note, when all the patients were included, the radiological objective response was still more frequent in the low MGMT group with a cutoff at either $50(P=0.045)$ or $100(P=0.014)$.

\section{MGMT predictive value of prolonged stabilization}

The percentage of patients who did not progress 24 months after the treatment initiation decreased with the rise of
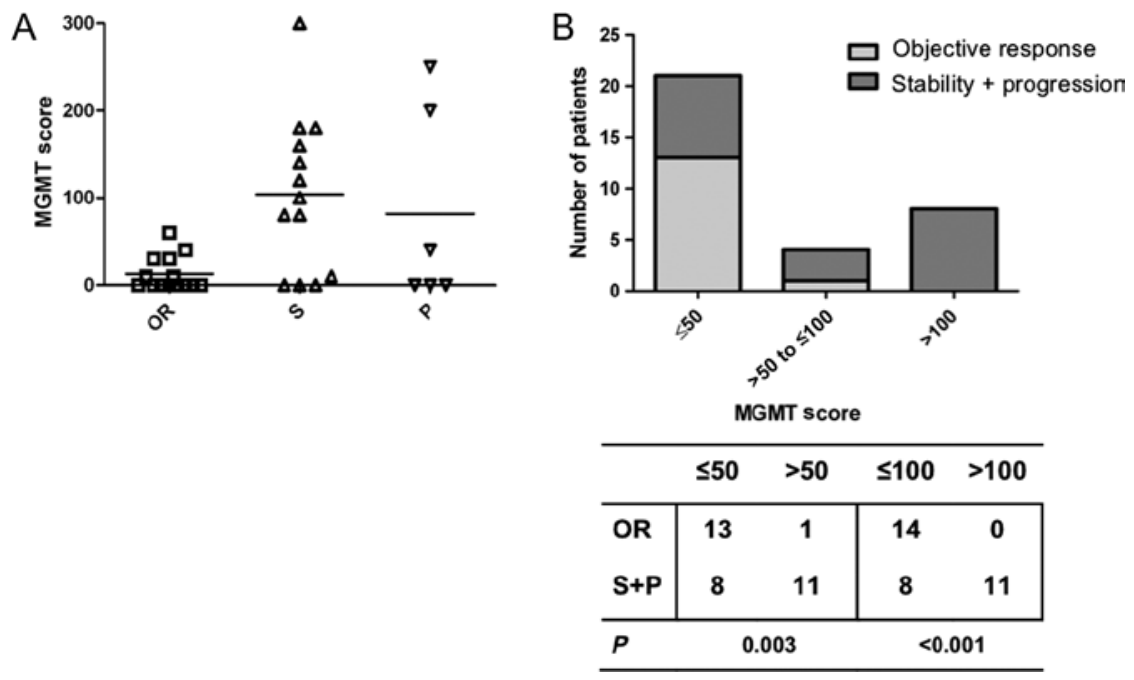

Figure 3

Radiological response according to immunohistochemical expression of MGMT. (A) MGMT scores according to the best radiological response $(\mathrm{OR}$, objective response; $\mathrm{P}$, progression; $\mathrm{S}$, stabilization); (B) repartition of patients within the responder $(\mathrm{OR})$ or nonresponder $(\mathrm{S}+\mathrm{P})$ groups according to their MGMT expression (low: $\leq 50$, intermediate: $>50$ to $\leq 100$, and high: $>100$ ). 
Table 3 Proportion of patients with no progression according to time and MGMT immunohistochemical expression.

\begin{tabular}{|c|c|c|c|c|c|c|}
\hline \multirow{2}{*}{$\begin{array}{l}\text { Percentage of } \\
\text { patients that did } \\
\text { not progress at: }\end{array}$} & \multicolumn{6}{|c|}{ Different cutoffs of MGMT score } \\
\hline & $\leq 50$ & $>50$ & $\leq 100$ & $>100$ & $\leq 150$ & $>150$ \\
\hline 6 months & $81 \%$ & $74 \%$ & $83 \%$ & $62 \%$ & $85 \%$ & $50 \%$ \\
\hline 12 months & $81 \%$ & $60 \%$ & $79 \%$ & $50 \%$ & $81 \%$ & $33 \%$ \\
\hline 18 months & $75 \%$ & $51 \%$ & $74 \%$ & $37 \%$ & $70 \%$ & $33 \%$ \\
\hline 24 months & $65 \%$ & $0 \%$ & $53 \%$ & $0 \%$ & $45 \%$ & $0 \%$ \\
\hline$P$ & \multicolumn{2}{|c|}{0.009} & \multicolumn{2}{|c|}{0.008} & \multicolumn{2}{|c|}{0.004} \\
\hline
\end{tabular}

For each threshold, the left column refers to the percentage of nonprogression in the patient group with an MGMT score below the threshold and the right column refers to the percentage of nonprogression in the patient group with an MGMT score above the threshold.

the MGMT score threshold from 50 to 100 but remained significantly higher in the groups of patients with a lower MGMT (Table 3). For instance, $65 \%$ of patients with an MGMT score below 50 had not progressed at 24 months, whereas none with an MGMT score above 50 . This rate of no progression at 24 months slightly decreased with an MGMT score threshold set at 100 (53 vs 0\% in patients with an MGMT score below or above 100, respectively). However, the rates of patients who did not progress after 18 months of treatment were fairly similar to patients with an MGMT score $<50$ or $<100$ (75 and 74\%, respectively) (Table 3 ). Similar results were obtained with more stringent cutoffs $(15,30)$ (Supplementary Fig. 2D, E, and F). The rate of nonprogression according to time was not significantly different with a cutoff set at 0 .

\section{Characteristics of nonresponders}

The median MGMT score was low in the objective response group (0 (0-60)) and higher in the group with only tumor stabilization (80 (0-300)). Unexpectedly, the median MGMT score in nonresponder patients was low (15 (0-250)). Six out of the eight progressive patients had an MGMT score below 50. To explore this discrepancy, we searched for factors that could explain TEM resistance despite a low MGMT status. The median Ki-67 was significantly higher in the nonresponder group (17.5\% (1-40)) compared with the group achieving tumor control $(8 \%(1-12)$ in patients with stable disease and 5.5\% (2-20) in patients with an objective response; $P=0.01$ ). Six out of the eight nonresponder patients had a Ki- $67 \geq 10 \%$ and five out of eight a Ki- $67 \geq 15 \%$. In the whole population, seven patients had a Ki- $67 \geq 15 \%$. Among them, two had an objective response and five a tumor progression.

A functional MMR system is required for TEMinduced cell toxicity (Zhang et al. 2011). We, therefore, hypothesized that nonresponders (especially those with a low MGMT expression) may have a defective MMR system, resulting in a microsatellite instable status. We assessed the microsatellite status in seven out of the eight nonresponders. All had a microsatellite stable status.

\section{Predictive value of MGMT promoter methylation}

The methylation of four CpGs within the exon 1 reported to have a predictive value in TEM-treated glioblastoma was assessed by pyrosequencing (Quillien et al. 2012). MGMT promoter methylation could be assessed in 29 patients. For two patients, the extracted DNA was repeatedly of too poor quality to be used, and the rest of the samples were either FNA biopsies with tumor cells too diluted within inflammatory/normal cells or liver biopsies with too few tumor cells. MGMT promoter methylation tended to be higher in the group with low MGMT $(\leq 100)(P=0.08)$, and a trend toward a higher methylation was observed in the group of patients that presented an objective response $(P=0.1)$. With the promoter methylation cutoff used in glioblastoma studies (7\%), patients with a

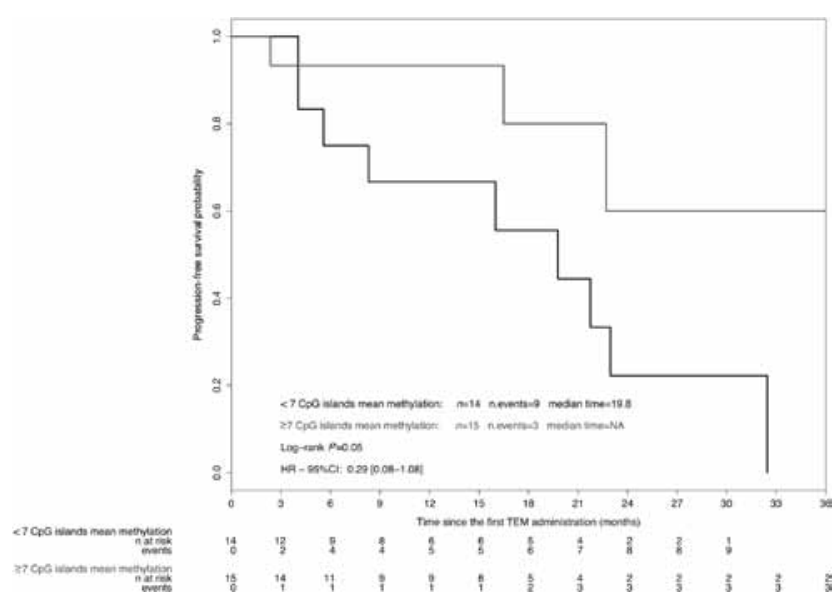

Figure 4

PFS in patients receiving TEM-based therapy for WDPNET with an MGMT promoter methylation above or below $7 \%$.

Published by Bioscientifica Ltd. 
hypermethylated promoter had a longer PFS $(\mathrm{HR}=0.29$ $(0.08-1.08), P=0.05)$ (Fig. 4).

\section{Discussion}

In this study, we assessed retrospectively the predictive value of MGMT expression by immunohistochemistry and its promoter methylation by pyrosequencing in a cohort of well-differentiated PNETs treated by TEMbased chemotherapy. Both MGMT expression and MGMT promoter methylation influenced PFS. Although an objective response was obtained quasi-exclusively in tumors with a very low MGMT expression (score $\leq 50$ ), prolonged stabilizations were seen in tumors with an intermediate level of expression (score between 50 and 100).

The prognostic and predictive values of MGMT are still controversial, mainly because different nonequivalent techniques were used to assess the MGMT status together with small and/or heterogeneous cohorts (Ekeblad et al. 2007, Kulke et al. 2009, Schmitt et al. 2014, Walter et al. 2015). The strengths of this study are the homogeneity of the population, especially the primary tumor location and type of chemotherapy regimen, and its size. In addition, most tumors were progressive at the introduction of TEM, which reflects well the true clinical practice. Fifteen out of forty-three patients were treated with capecitabine in addition to TEM. Although the number of patient was too low to assess a possible effect of capecitabine on the MGMT predictive value, no biological link has been described between them.

The quantification of a biomarker expression by immunochemistry may be difficult. It requires major efforts in standardization. Hence, significant differences can be observed using different antibodies and staining procedures/evaluation. We used the same MGMT clone (MT3.1) as in the two largest studies, and all staining procedures were performed on a calibrated diagnostic automat, thus reducing some of the staining variability (Kulke et al. 2009, Schmitt et al. 2014). The reproducibility of the scoring between the pathologists that graded the slides was excellent $(\kappa=0.85)$. MGMT expression can be heterogeneous within a tumor (intensity of the number of expressing cells); thus, it appears to be best evaluated on whole slides rather than tissue microarrays. All previous studies used a low cutoff of 0,5 , or $10 \%$ of MGMTexpressing cells to dichotomize tumors, and the intensity of staining was not taken into account (Ekeblad et al. 2007, Kulke et al. 2009, Schmitt et al. 2014, Walter et al. 2015).
We favored a composite and continuous score to better assess both tumor heterogeneity and the relationship between MGMT expression and clinical response in patients who received TEM-based chemotherapy. Using this approach, we were able to define different thresholds of MGMT scores adapted to the clinical goal. Overall, an objective response can reasonably be achieved only in very low-expressing tumor (MGMT score $\leq 50$ ) (Table 3). This is in accordance with most reports, but our score appears more flexible than those with a single cutoff point. Patients' selection should, therefore, be strict in neoadjuvant setting, because maximal volume reduction of a large tumor is the main objective of chemotherapy with the aim to propose surgical resection thereafter. This approach also allowed us to quantify the rate of nonprogressive patients at 6-month intervals after TEM initiation for several MGMT thresholds (Table 3). The percentage of patients whose disease was still controlled at 18 months remained valuable when the MGMT threshold was set at 100, although it dropped frankly above. Although no objective response was obtained in patients with such an intermediate MGMT expression, prolonged tumor control was obtained in patients who would have been otherwise not eligible with a strict positive/negative grading scheme.

Although all patients that did respond had a low MGMT expression, some patients with an MGMT score of 0 did not respond to TEM. This explains why with a strict negative/positive cutoff, the MGMT expression is not associated with the PFS. If the threshold is slightly raised to 15 or 30 , these discordant patients are outnumbered by the patients with a low MGMT score and a good response, and the MGMT expression is associated with the PFS. To provoke TEM-induced DNA damages and kill tumor cells requires a functional MMR system in addition to low MGMT expression (Friedman et al. 1998, Zhang et al. 2011). None of our low MGMT nonresponder patients had a microsatellite instability phenotype. The base excision repair (BER), another DNA repair system, could contribute to TEM resistance similarly to MGMT. We were not able to test this hypothesis, as there is no available routine test to assess BER functionality, especially in FFPE samples. This suggests that additional biomarkers are necessary to identify these low MGMT-resistant patients. The single particularity of some resistant patients was a high Ki-67 index $(5 / 8,>15 \%)$. Welin and coworkers reported that TEM-CAP could induce partial response in highly proliferative poorly differentially carcinomas (Welin et al. 2011). This suggests that although TEM could be 
efficient in proliferative G3 PNET, MGMT expression by immunochemistry may not be predictive in this tumor type. Our current series cannot firmly validate this hypothesis due to the limited number of patients evaluated.

Reports about non-NETs suggested that the predictive value of MGMT promoter methylation is superior to that of its expression by immunochemistry (Karayan-Tapon et al. 2010, Kohsaka et al. 2012, Kreth et al. 2013). Several techniques have been compared with assess MGMT promoter methylation and pyrosequencing on four CpGs in exon 1 appears to be the most robust and predictive manner in glioblastoma and PNET (Karayan-Tapon et al. 2010, Quillien et al. 2012, Schmitt et al. 2014, Walter et al. 2015). We, therefore, used this technique and also found that increased promoter methylation was associated with low MGMT protein expression, although it was not perfectly correlated at the individual level. This discrepancy is not entirely surprising, as numerous methylation-independent mechanisms of MGMT expression exist, such as miRNA, histone modifications, or NF-kB/STAT3-dependent regulation (Lavon et al. 2007, Ramakrishnan et al. 2011, Kitange et al. 2012, Kohsaka et al. 2012, Zhang et al. 2012, Kreth et al. 2013). In addition, comparative CpG arrays and transcriptomic arrays suggested that the CpGs tested in the pyrosequencing assay are not the ones most associated with MGMT mRNA expression (Bady et al. 2012). Yet although increased promoter methylation was not significantly associated with $\mathrm{OR}$, it was significantly associated in our study with a longer PFS under TEM treatment.

The predictive value of pyrosequencing and immunochemistry cannot be properly compared in our study, but several publications have suggested that MGMT promoter methylation also has an intrinsic prognostic value in glioblastoma and PNET (Karayan-Tapon et al. 2010, Christians et al. 2012, Schmitt et al. 2014, Walter et al. 2015). This prognostic value probably does not lie solely in the MGMT promoter methylation but is rather a surrogate marker of a global CIMP-like phenotype (Bady et al. 2012, Walter et al. 2015). In addition, MGMT promoter methylation is difficult or impossible to assess in FNA biopsies or liver biopsies if the tumor cell count is too low or the tumor cells largely outnumbered by nontumor cells. Although the immunochemistry assessment in such conditions may imperfectly reflect the tumor heterogeneity, it may still prove to be useful to propose an alternative therapy to a patient with a very high MGMT score.

In conclusion, low MGMT expression assessed by immunochemistry in TEM-treated patients with PNETs is associated with radiological objective response and increased PFS. The evaluation of MGMT expression by immunochemistry is cost effective and routinely feasible. A continuous score may help select additional patients with an intermediate expression that may still achieve a prolonged stabilization under TEM.

\section{Supplementary data}

This is linked to the online version of the paper at http://dx.doi.org/10.1530/ ERC-16-0117.

\section{Declaration of interest}

The authors declare that there is no conflict of interest that could be perceived as prejudicing the impartiality of the research reported.

\section{Funding}

This research did not receive any specific grant from any funding agency in the public, commercial or not-for-profit sector.

\section{References}

Bady P, Sciuscio D, Diserens A-C, Bloch J, van den Bent MJ, Marosi C, Dietrich P-Y, Weller M, Mariani L, Heppner FL, et al. 2012 MGMT methylation analysis of glioblastoma on the Infinium methylation BeadChip identifies two distinct CpG regions associated with gene silencing and outcome, yielding a prediction model for comparisons across datasets, tumor grades, and CIMP-status. Acta Neuropathologica 124 547-560. (doi:10.1007/s00401-012-1016-2)

Chan JA, Stuart K, Earle CC, Clark JW, Bhargava P, Miksad R, Blaszkowsky L, Enzinger PC, Meyerhardt JA, Zheng H, et al. 2012 Prospective study of bevacizumab plus temozolomide in patients with advanced neuroendocrine tumors. Journal of Clinical Oncology 30 2963-2968. (doi:10.1200/JCO.2011.40.3147)

Christians A, Hartmann C, Benner A, Meyer J, von Deimling A, Weller M, Wick W \& Weiler M 2012 Prognostic value of three different methods of MGMT promoter methylation analysis in a prospective trial on newly diagnosed glioblastoma. PLOS ONE 7 e33449. (doi:10.1371/journal.pone.0033449)

Cohen J 1968 Weighted kappa: nominal scale agreement with provision for scaled disagreement or partial credit. Psychological Bulletin 70 213-220. (doi:10.1037/h0026256)

Durante C, Boukheris H, Dromain C, Duvillard P, Leboulleux S, Elias D, de Baere T, Malka D, Lumbroso J, Guigay J, et al. 2009 Prognostic factors influencing survival from metastatic (stage IV) gastroenteropancreatic well-differentiated endocrine carcinoma. Endocrine-Related Cancer 16 585-597. (doi:10.1677/ERC-08-0301)

Eisenhauer EA, Therasse P, Bogaerts J, Schwartz LH, Sargent D, Ford R, Dancey J, Arbuck S, Gwyther S, Mooney M, et al. 2009 New response evaluation criteria in solid tumours: revised RECIST guideline (version 1.1). European Journal of Cancer 45 228-247. (doi:10.1016/j. ejca.2008.10.026)

Ekeblad S, Sundin A, Janson ET, Welin S, Granberg D, Kindmark H, Dunder K, Kozlovacki G, Orlefors H, Sigurd M, et al. 2007 Temozolomide as monotherapy is effective in treatment of advanced malignant neuroendocrine tumors. Clinical Cancer Research $\mathbf{1 3}$ 2986-2991. (doi:10.1158/1078-0432.CCR-06-2053)

Fine RL, Gulati AP, Krantz BA, Moss RA, Schreibman S, Tsushima DA, Mowatt KB, Dinnen RD, Mao Y, Stevens PD, et al. 2013 Capecitabine and temozolomide (CAPTEM) for metastatic, well-differentiated 
neuroendocrine cancers: the Pancreas Center at Columbia University experience. Cancer Chemotherapy and Pharmacology 71 663-670. (doi:10.1007/s00280-012-2055-z)

Friedman HS, McLendon RE, Kerby T, Dugan M, Bigner SH, Henry AJ, Ashley DM, Krischer J, Lovell S, Rasheed K, et al. 1998 DNA mismatch repair and O6-alkylguanine-DNA alkyltransferase analysis and response to Temodal in newly diagnosed malignant glioma. Journal of Clinical Oncology 16 3851-3857.

Hegi ME, Diserens A-C, Gorlia T, Hamou M-F, de Tribolet N, Weller M, Kros JM, Hainfellner JA, Mason W, Mariani L, et al. 2005 MGMT gene silencing and benefit from temozolomide in glioblastoma. New England Journal of Medicine 352 997-1003. (doi:10.1056/NEJMoa043331)

Karayan-Tapon L, Quillien V, Guilhot J, Wager M, Fromont G, Saikali S, Etcheverry A, Hamlat A, Loussouarn D, Campion L, et al. 2010 Prognostic value of O6-methylguanine-DNA methyltransferase status in glioblastoma patients, assessed by five different methods. Journal of Neuro-Oncology 97 311-322. (doi:10.1007/s11060-009-0031-1)

Kitange GJ, Mladek AC, Carlson BL, Schroeder MA, Pokorny JL, Cen L, Decker PA, Wu W, Lomberk GA, Gupta SK, et al. 2012 Inhibition of histone deacetylation potentiates the evolution of acquired temozolomide resistance linked to MGMT upregulation in glioblastoma xenografts. Clinical Cancer Research 18 4070-4079. (doi:10.1158/1078-0432.CCR-12-0560)

Kohsaka S, Wang L, Yachi K, Mahabir R, Narita T, Itoh T, Tanino M, Kimura T, Nishihara H \& Tanaka S 2012 STAT3 inhibition overcomes temozolomide resistance in glioblastoma by downregulating MGMT expression. Molecular Cancer Therapeutics 11 1289-1299. (doi:10.1158/1535-7163.MCT-11-0801)

Kreth S, Limbeck E, Hinske LC, Schütz SV, Thon N, Hoefig K, Egensperger R \& Kreth FW 2013 In human glioblastomas transcript elongation by alternative polyadenylation and miRNA targeting is a potent mechanism of MGMT silencing. Acta Neuropathologica 125 671-681. (doi:10.1007/s00401-013-1081-1)

Kulke MH, Stuart K, Enzinger PC, Ryan DP, Clark JW, Muzikansky A, Vincitore M, Michelini A \& Fuchs CS 2006 Phase II study of temozolomide and thalidomide in patients with metastatic neuroendocrine tumors. Journal of Clinical Oncology 24 401-406. (doi:10.1200/JCO.2005.03.6046)

Kulke MH, Hornick JL, Frauenhoffer C, Hooshmand S, Ryan DP, Enzinger PC, Meyerhardt JA, Clark JW, Stuart K, Fuchs CS, et al. 2009 O6-methylguanine DNA methyltransferase deficiency and response to temozolomide-based therapy in patients with neuroendocrine tumors. Clinical Cancer Research 15 338-345. (doi:10.1158/1078-0432.CCR-08-1476)

Lavon I, Fuchs D, Zrihan D, Efroni G, Zelikovitch B, Fellig Y \& Siegal T 2007 Novel mechanism whereby nuclear factor kappaB mediates DNA damage repair through regulation of O(6)-methylguanine-DNAmethyltransferase. Cancer Research 67 8952-8959. (doi:10.1158/00085472.CAN-06-3820)

Olsen IH, Sørensen JB, Federspiel B, Kjaer A, Hansen CP, Knigge U \& Langer SW 2012 Temozolomide as second or third line treatment of patients with neuroendocrine carcinomas. Scientific World Journal 2012 170496. (doi:10.1100/2012/170496)

Pavel M, Baudin E, Couvelard A, Krenning E, Öberg K, Steinmüller T, Anlauf M, Wiedenmann B \& Salazar R 2012 ENETS Consensus Guidelines for the management of patients with liver and other distant metastases from neuroendocrine neoplasms of foregut, midgut, hindgut, and unknown primary. Neuroendocrinology 95 157-176. (doi:10.1159/000335597)

Quillien V, Lavenu A, Karayan-Tapon L, Carpentier C, Labussière M, Lesimple T, Chinot O, Wager M, Honnorat J, Saikali S, et al. 2012
Comparative assessment of 5 methods (methylation-specific polymerase chain reaction, methylight, pyrosequencing, methylationsensitive high-resolution melting, and immunohistochemistry) to analyze O6-methylguanine-DNA-methyltranferase in a series of 100 glioblastoma patients. Cancer 118 4201-4211. (doi:10.1002/cncr.27392)

Ramakrishnan V, Kushwaha D, Koay DC, Reddy H, Mao Y, Zhou L, Ng K, Zinn P, Carter B \& Chen CC 2011 Post-transcriptional regulation of $\mathrm{O}(6)$-methylguanine-DNA methyltransferase MGMT in glioblastomas. Cancer Biomarkers: Section A of Disease Markers 10 185-193. (doi:10.3233/CBM-2012-0245)

Rindi G, Klöppel G, Alhman H, Caplin M, Couvelard A, de Herder WW, Erikssson B, Falchetti A, Falconi M, Komminoth P, et al. 2006 TNM staging of foregut (neuro)endocrine tumors: a consensus proposal including a grading system. Virchows Archiv 449 395-401. (doi:10.1007/s00428-006-0250-1)

Schmitt AM, Pavel M, Rudolph T, Dawsona H, Blank A, Komminoth P, Vassella E \& Perren A 2014 Prognostic and predictive roles of MGMT protein expression and promoter methylation in sporadic pancreatic neuroendocrine neoplasms. Neuroendocrinology 100 35-44. (doi:10.1159/000365514)

Schraml P, von Teichman A, Mihic-Probst D, Simcock M, Ochsenbein A, Dummer R, Michielin O, Seifert B, Schläppi M, Moch H, et al. 2012 Predictive value of the MGMT promoter methylation status in metastatic melanoma patients receiving first-line temozolomide plus bevacizumab in the trial SAKK 50/07. Oncology Reports 28 654-658. (doi:10.3892/or.2012.1826)

Skinazi F, Zins M, Menu Y, Bernades P \& Ruszniewski P 1996 Liver metastases of digestive endocrine tumours: natural history and response to medical treatment. European Journal of Gastroenterology \& Hepatology 8 673-678.

Strosberg JR, Fine RL, Choi J, Nasir A, Coppola D, Chen D-T, Helm J \& Kvols L 2011 First-line chemotherapy with capecitabine and temozolomide in patients with metastatic pancreatic endocrine carcinomas. Cancer 117 268-275. (doi:10.1002/cncr.25425)

Uno M, Oba-Shinjo SM, Camargo AA, Moura RP, de Aguiar PH, Cabrera HN, Begnami M, Rosemberg S, Teixeira MJ \& Marie SKN 2011 Correlation of MGMT promoter methylation status with gene and protein expression levels in glioblastoma. Clinics 66 1747-1755. (doi:10.1590/S1807-59322011001000013)

Walter T, van Brakel B, Vercherat C, Hervieu V, Forestier J, Chayvialle J-A, Molin Y, Lombard-Bohas C, Joly M-O \& Scoazec J-Y $2015 \mathrm{O}(6)-$ Methylguanine-DNA methyltransferase status in neuroendocrine tumours: prognostic relevance and association with response to alkylating agents. British Journal of Cancer 112 523-531. (doi:10.1038/bjc.2014.660)

Welin S, Sorbye H, Sebjornsen S, Knappskog S, Busch C \& Oberg K 2011 Clinical effect of temozolomide-based chemotherapy in poorly differentiated endocrine carcinoma after progression on first-line chemotherapy. Cancer 117 4617-4622. (doi:10.1002/cncr.26124)

Wong YF, Cheung TH, Lo KWK, Yim SF, Chan LKY, Buhard O, Duval A, Chung TKH \& Hamelin R 2006 Detection of microsatellite instability in endometrial cancer: advantages of a panel of five mononucleotide repeats over the National Cancer Institute panel of markers. Carcinogenesis 27 951-955. (doi:10.1093/carcin/bgi333)

Zhang J, Stevens MF \& Bradshaw TD 2011 Temozolomide: mechanisms of action, repair and resistance. Current Molecular Pharmacology 5 102-114. (doi:10.2174/1874467211205010102)

Zhang W, Zhang J, Hoadley K, Kushwaha D, Ramakrishnan V, Li S, Kang C, You Y, Jiang C, Song SW, et al. 2012 miR-181d: a predictive glioblastoma biomarker that downregulates MGMT expression. Neuro-Oncology 14 712-719. (doi:10.1093/neuonc/nos089)

Received in final form 9 June 2016

Accepted 28 June 2016

Accepted Preprint published online 28 June 2016 http://erc.endocrinology-journals.org

DOI: 10.1530/ERC-16-0117 (c) 2016 Society for Endocrinology Printed in Great Britain
Published by Bioscientifica Ltd 